\title{
Improving the principles of short-term electric load forecasting of the Irkutsk region
}

\author{
Vladimir Kornilov ${ }^{1}$, Victor Kurbatsky ${ }^{2}$, and Nikita Tomin ${ }^{2}$ \\ ${ }^{1}$ JSC “SO UPS” Irkutsk Regional Dispatch Office (RDO), Irkutsk, Russia \\ ${ }^{2}$ Melentiev Energy Systems Institute, 130 Lermontov str., Irkutsk, Russia
}

\begin{abstract}
Forecasting of electric load (EL) is an important task for both electric power entities and large consumers of electricity [1]. Large consumers are faced with the need to compose applications for the planned volume of EL, and the deviation of subsequent real consumption from previously announced leads to the appearance of penalties from the wholesale market. In turn, electricity producers are interested in forecasting the demand for electricity for prompt response to its fluctuations and for the purpose of optimal infrastructure development. The most difficult and urgent task is the hourly forecasting of EL, which is extremely important for the successful solution of problems of optimization of generating capacities, minimization of power losses, dispatching control, security assessment of power supply, etc. Ultimately, such forecasts allow optimizing the cash costs for electricity and fuel or water consumption during generation. This paper analyzes the experience of the branch of JSC "SO UPS" Irkutsk Regional Dispatch Office of the procedure for short-term forecasting of the EL of the Irkutsk region.
\end{abstract}

\section{The existing functional scheme for forecasting}

At present, this task is carried out with the use of the corporate software "Software of the hierarchical forecasting system for planning and scheduling of regimes" (HSP), developed by Energostat. [2]

The first step is the launch of the statistical forecast module. As a result of the module work, EL predictions are generated for the period of the corresponding planning cycle. The following models of statistical forecasting are used in HSP software:

- HSP forecasting model taking into account seasonality and weather factors.

- model of the previous average day, taking into account meteorological factors;

At the same time, the forecast is carried out taking into account the forecast of meteorological factors obtained from the Hydrometeorological Center of Russia, and if they are not available, then a statistical forecast model of meteorological factors based on the seasonal temperature curve and the latest actual data is used for their formation. In each branch of RDO of JSC "SO UES" forecasting is carried out for each territory of the operational area of the given branch and all areas of forecasting of subordinate branches (up to power areas of power systems).

The forecasting models are set up by the developer who, according to certain methods, makes changes in the tuning coefficients of the forecast models, depending on the predicted temperature of the ambient air. Earlier, classical techniques for daily average temperatures were used, when the average daily temperature was averaged over the last 3 days: for the autumn-winter period (AWP) with weights $0.5,0.3,0.2$; for summer - with weights 0.7 , $0.2,0.1$. However, this weighted classical averaging of the average daily and hourly temperatures does not accurately simulate the dynamics under sudden temperature fluctuations. Proceeding from this, there are difficulties in modeling processes in hot weather, when it is necessary to take into account the fact of long-term preservation of high temperatures of outside air. This requires the use of large values of the lag parameter. At the same time, there are situations when it is necessary to instantly increase the conditioning loads (at temperatures above 30 degrees), which already requires a low lag parameter value. In addition, in hot weather, cloudiness can lower EL, because conditions for cooling the radiators of air conditioners improve.

According to the developer's technique, the tuning factors are selected from the condition of providing the best forecasting accuracy. The temperature delay parameters are defined for the AWP - 23 hours, for the summer period - 5 hours. At the same time, a decrease in the delay in the effect of temperature in the currently used models can lead to a decrease in the overall accuracy of the prediction. On certain days the forecast will be fulfilled perfectly, but on other days it can be much worse.

\section{Forecast of hourly EL of Irkutsk region}

Historically, the forecasted hourly EL of the Irkutsk region per day X-1 (i.e., one day before the start of the 
operational day) is defined as the sum of the forecasts of three major energy areas: Irkutsk-Cheremkhovo, BratskUst-Ilimsk and Bodaybo (Figure 1). For hourly forecasting of EL of these regions, there is a fairly large array of archival information, on the basis of which automated prediction models having sufficient accuracy have been created.

As can be seen in Fig. 2., the predictive models used based on the "seasonal curves" method [17] give a good forecast accuracy, but there are certain shortcomings revealed during the daily use of the method used. This is particularly pronounced in the unsatisfactory operation of models with sufficiently sharp and significant temperature changes, with a deviation from the average statistical values in the days in question.

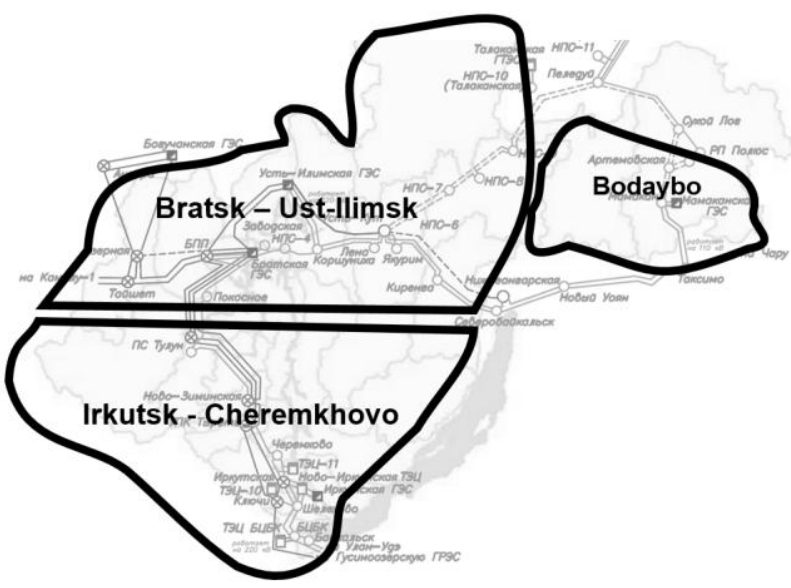

Fig. 1. Major energy areas of Irkutsk region

The annual change in the EL values of these regions for 2016 is shown in Fig. 2.

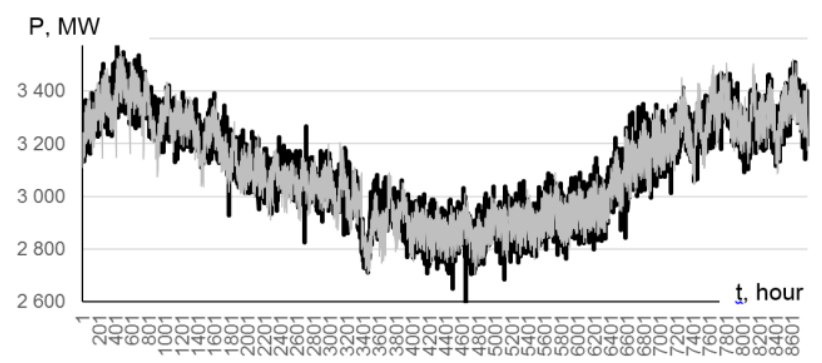

—Fact AutoForecast

a. Hourly load of Bratsk-Ust-Ilimsk area

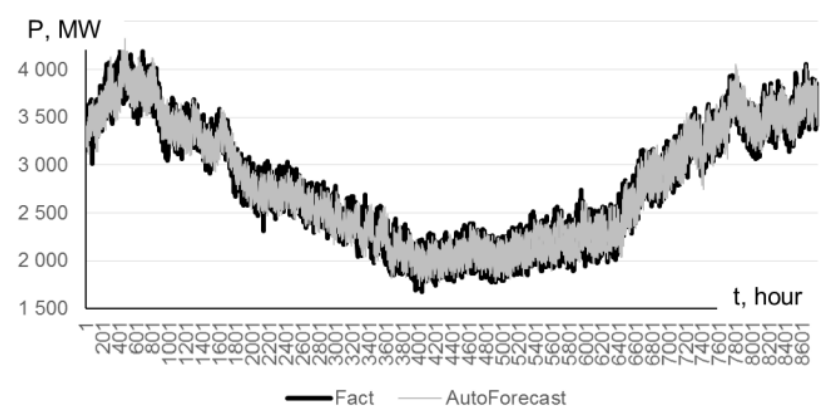

b. Hourly load of Irkutsk-Cheremkhovo area

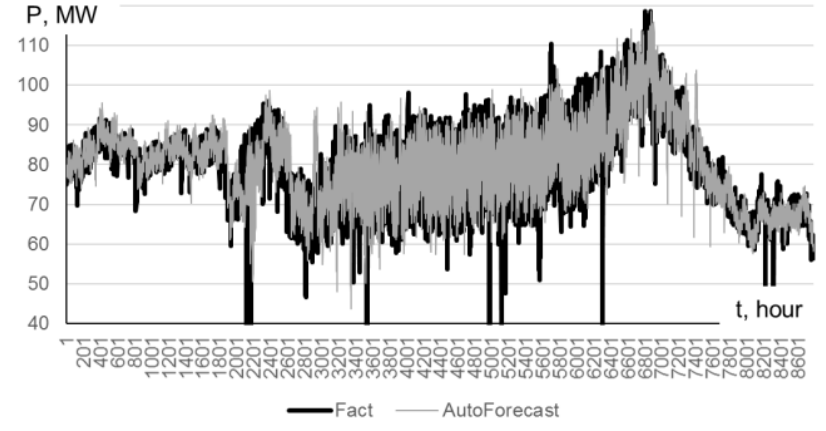

c. Hourly load of Bodaybo area

Fig. 2. Annual EL graphs of Irkutsk region

The method of "seasonal curves", based on the correlation and regression analysis of data, allows to analytically describe the fluctuations in the load and weather factors in the during of the year. The method assumes additivity of the load:

$$
P(i)=P_{0}(i)+P_{\text {season }}(i)+\delta P(i)+\gamma P(i),
$$

where $\mathrm{i}$ - hour of the day (1-24); P(i) is the actual load of the power system; $\mathrm{P}_{0}(\mathrm{i})$ - the basic component, determined by stable production cycles, daily and weekly uneven load schedule; $\mathrm{P}_{\text {season }}(\mathrm{i})$ - seasonal component (seasonal curve), determined by seasonal fluctuations in the load during of the year. This component is due, first of all, to the deep seasonal fluctuations of meteorological factors - temperature and illumination; $\delta \mathrm{P}(\mathrm{i})$ the component determined by irregular fluctuations of meteofactors (irregular deviations of meteofactors from stable seasonal cycles are considered irregular); $2 \mathrm{P}(\mathrm{i})$ - residual component, determined by the influence of unaccounted factors.

To exclude the effect of random emissions and to obtain a mathematical model of the seasonal component, $\mathrm{P}_{\text {season }}$, are approximated by Fourier series:

$$
P_{\text {season }}(n)=\frac{A_{0}}{2}+\sum_{k=1}^{N}\left(A_{k} \cos k \omega n+A_{k}^{\prime} \sin k \omega n\right),
$$

where $A_{o}, A_{k}, A_{k}^{\prime}$ are the Fourier coefficients; $\omega=\frac{2 \pi}{T}$;

$\mathrm{T}=365 ; \mathrm{N}$ is the number of terms in the Fourier series.

The coefficients are estimated using the method of least squares. The order $\mathrm{N}$ is determined experimentally by a gradual increase in the order of the polynomial. As a criterion for stopping the increase in degree is checking the variances at each step. The result of the calculations is the matrix of Fourier coefficients of dimension $(25 \bullet(2 \mathrm{~N}$ 1) 7 ). In practice, the optimal degree of polynomial for describing the seasonal curve for different hours of the day ranges from 4 to 15 .

\section{Case Study}

Let's consider the model of the EL forecast for August 10, 2016. The peculiarity of these days was a significant and sharp decrease in the temperature of the surrounding air. At the same time, the decrease in temperature was not predicted with sufficient accuracy by the supplier of 
temperature forecasts (Fig. 3). In Fig. 4 shows the temperature change in Irkutsk by the hours of August 2016, also confirming an abnormal temperature change.

In Fig. 5 shows the results of the work of forecast models. It can be seen that the increase in EL was not predicted with sufficient accuracy. The forecast of the EL, performed on the forecasted temperature for one district, gave deviations of up to $3.75 \%$ (in the Forecast 1 chart). At the same time, the forecast made on the next day,
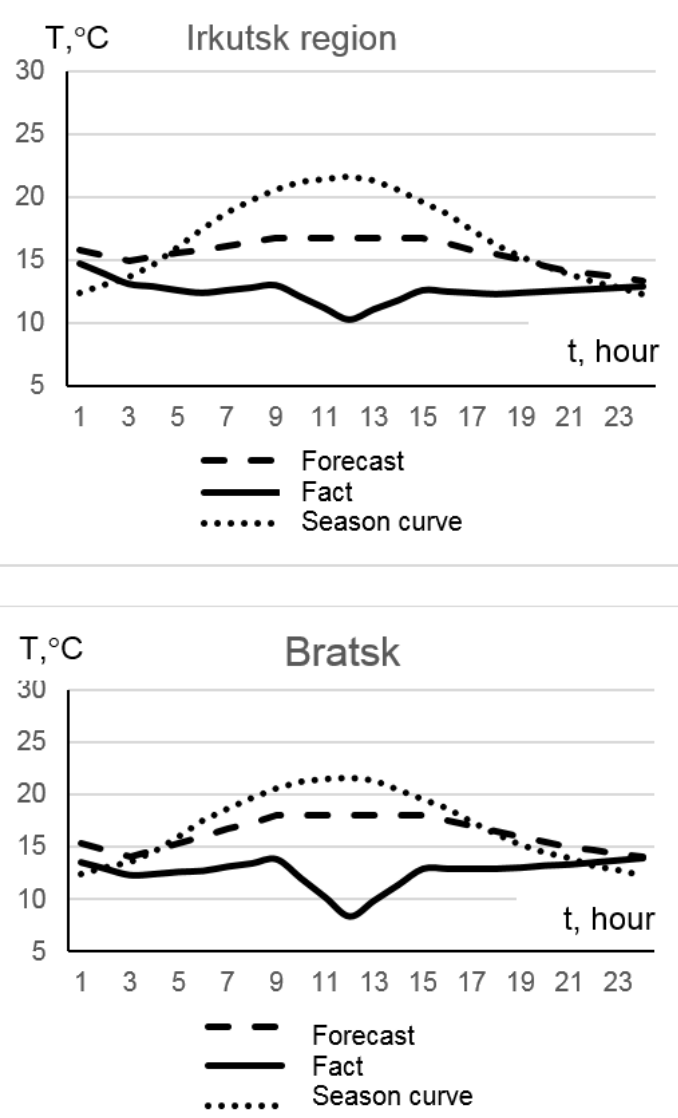

using the actual temperature, increased the deviation to $4.95 \%$ (in the Forecast $1+1$ chart). The forecast of the EL performed on the predicted temperature in three regions gave deviations to $4.22 \%$ (in the Forecast 3 chart). In turn, the forecast made the next day using the actual temperature increased the deviation to $4.42 \%$ (in the Forecast $3+1$ chart).
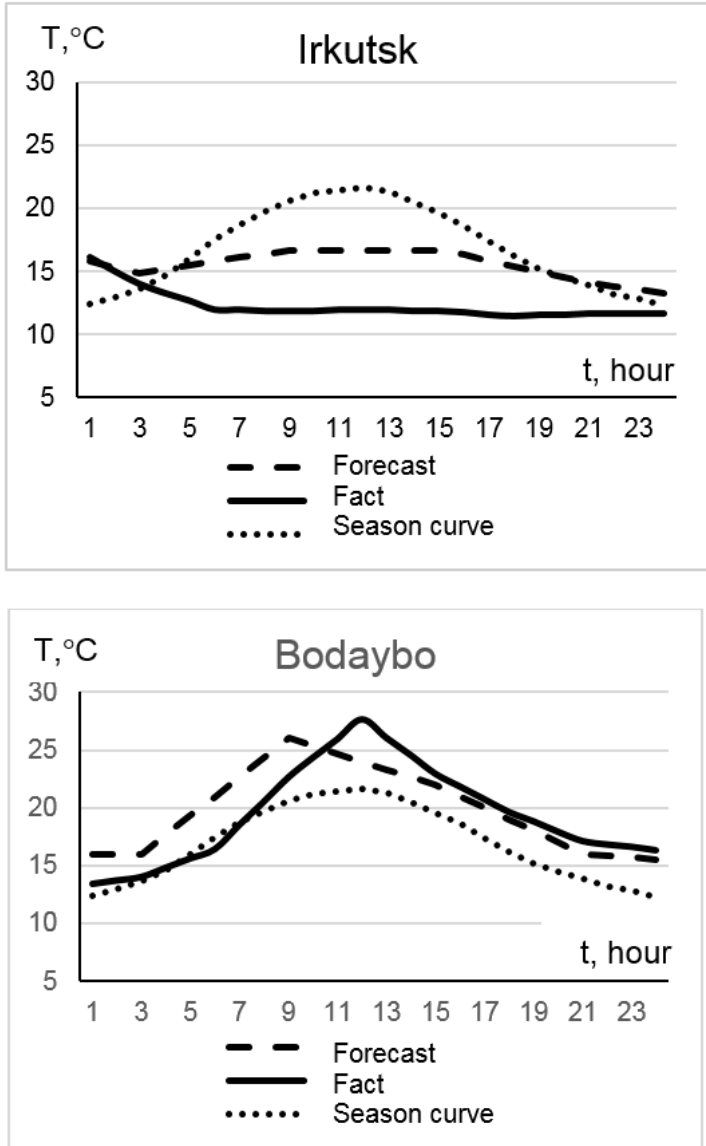

Fig. 3. Forecasts, facts and seasonal temperatures of 10 august 2016

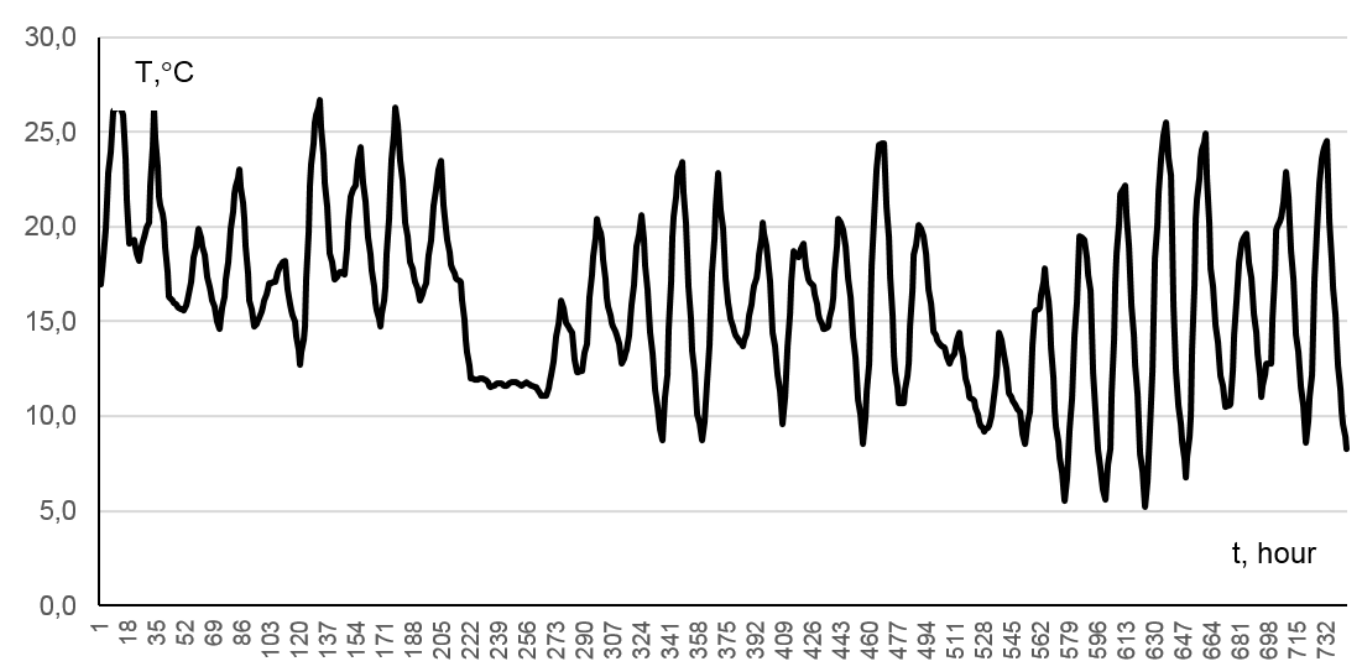

Fig. 4. Temperature in Irkutsk in august 2016, hourly 


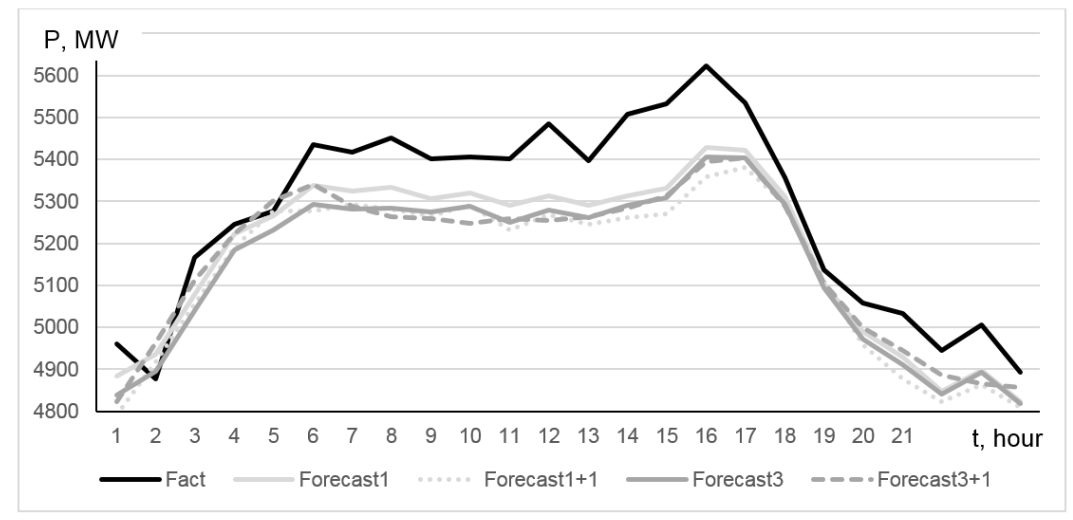

Fig. 5. The results of the forecast models

From the example, it is evident that the currently accepted "boundary" value of 4\% accuracy is not always achievable with existing models and the forecasting system. The problem is worse by the fact that the settings that affect the accuracy of forecasting the model parameters are currently "closed" for the direct specialist who performs the EL predictions. At the same time, an adequate correction of the temperature forecast from the supplier presents a certain problem and is not realized at the moment by the standard procedures of the software used.

Then, in the existing mathematical model, a number of problems have not been solved, which are partially described above. Therefore, at the present moment, the task arises to improve the performance of forecasting functions for hourly EL.

\section{Consideration of alternative methods for predicting EP}

Existing market management principles, first of all the current rules of operation in the wholesale electric power and capacity market [3], determine the special significance of mathematical and software models for short-term forecasting of EP. As a rule, a deviation in EL of more than $4 \%$ (depending on specific regulatory requirements [4]) from the planned leads to additional costs. At the same time, the situation is complicated by the need to link the forecasting method used to specific real conditions, including "irregular" effects (meteorological factors, holidays, bad data, etc.), which are often adjusted in "manual mode". As a result, specialists of various RDUs face the difficult task of compiling a reliable EL forecast.

Despite the commonality of the task of short-term EL forecasting facing each energy system, there are many different methods in the scientific literature, most of which are a combination of different statistical approaches. These approaches can be divided into classical (deterministic, stochastic) and intellectual (algorithms of machine learning, expert systems, fuzzy logic). Deterministic methods are classical causal models of load and weather parameters (primarily ambient air temperatures). Such methods include curve fitting, data extrapolation, and smoothing methods [5-7]. Stochastic methods model the load behavior as a kind of stochastic process. This category includes: Kalman filtration, autoregressive integrated moving average, etc. [7, 8].

However, qualitative simulation of EL in modern conditions is a complex process due to nonlinear relationships between dependent factors and the load burdened by possible distortions and incompleteness of incoming data. Often these circumstances are an insurmountable obstacle to classical approaches. In connection with this, in recent years, methods of machine learning, such as artificial neural networks (ANN) [9-11], the support vector method (SVM) [12], decision trees [13] have been widely used in EL prediction tasks. Machine methods succeeded in overcoming these difficulties due to the best approximating abilities, primarily non-linear functions [9]. Their main difference from classical algorithmic approaches is that such models are taught by examples.

The prognosis model learns to "draw conclusions" without human help on the basis of the experience gained in the learning process and the original abilities to generalize (working with new data). The effectiveness of this approach for the task of short-term forecasting of EL is confirmed by a large number of studies $[14,15]$. In particular, on the example of the data of the branch of JSC "SO UPS" of the Rostov RDO, the ANN models gave errors of $3.04-4.78 \%$, the model of the SVM - 1.22$3.22 \%$ [16].

It is assumed that the newly developed forecasting system will not be a built-in HSP module. The new system can be used in parallel with HSP software. At the same time, the results of the new system will be for the performer to prognosticate an additional source of an alternative forecast of EL (including using alternative sources of ambient air temperature forecast). As a result, the contractor can manually change the final values of the predicted EL in the HSP software at the final stage.

\section{Further directions of research}

Proceeding from the mentioned problems of short-term forecasting of EL based on the HSP software, it is possible to single out several research directions for improving forecasts in the practice of the Irkutsk Regional Dispatch Office: 
1. Decomposition of EL areas on the basis of the analysis of the presence of telemetry and the possibility of fixing EL in more "shallow" areas of the Irkutsk region (use of telemetry data, use of Counters data, etc.), as well as additional verification of the temperature forecasts at the performer level.

2. Development of alternative forecasting models that allow automating the prediction process and obtaining more accurate predictions. An effective technology for this is machine learning, in particular, the algorithms of ANN and decision trees.

3. Analysis of the importance of using additional input data for forecasting (cloudiness, precipitation, wind, etc.)

\section{Conclusion}

1. The modern hierarchical forecasting system HSP in most cases has an acceptable accuracy of the EL forecast for the Irkutsk power system.

2. During the off-season periods, with significant deviations in ambient air temperature from the average annual, the forecast errors of existing models exceed $4 \%$.

3. It is advisable to conduct further research on the possibility of improving the accuracy of forecasting using machine learning techniques, in particular, ANN algorithms and decision trees, and to explore the possibility of applying additional input parameters for the performance of EL predictions.

\section{References}

1. M.M. Migranov, A.V. Melnikov ELECTRICITY. Transmission and distribution, №3, 60-64, (2017).

2. Software for a hierarchical system for predicting power consumption for planning the EEC regimes (HSP) Version 3.14 Volume 1. User's manual. (Edition of 01.10.2016) [Electronic resource], Moscow, (2016), CDROM.

3. Rules of the wholesale electricity and capacity market, http://www.consultant.ru/document/cons_ doc LAW 112537/

4. D. Singh, Jour. Electric Power Components and Systems. No. 30, 443-456, (2002).

5. W. R. Christiaanse, IEEE Trans. On Power Appar. Syst, PAS-3, 900-911, (1988).

6. A. D. Papalexopoulos, T. Hasterberg, IEEE Trans. On Power Systems, Vol.5, No.4, 1535-1544, (Nov. 1990).

7. S. R. Haung, IEE Proc. Gener. Trans. Distrib.,Vol. 144, No.5, 477-481, (Sept.1997).

8. H.S. Hippert, C. Eduardo Pedreira, R. Castro Souza, IEEE Trans. On Power Systems, Vol. 16. - No. 1, (2001).

9. G.P. Shumilova, N.E. Gotman, T.B. Startseva, Forecasting of electric loads in the operational management of electric power systems based on neural network structures (Ekaterinburg: UrB RAS, 2008).

10. V.G. Kurbatsky N.V Tomin, Electrical Engineering, No. 7., 26-32, (2006).

11. Nadtoka II, Al-Ziheri Balasim M. Modern problems of science and education. No. 6., 1-7. (2013).

12. Tomin N., Zhukov A., Sidorov D., Kurbatsky V., Panasetsky D., Spiryaev V. International Journal of
Artificial Intelligence), Vol. 13, No. 1, 211-228, (2015 Spring (March).

13. How Effective are Neural Networks at Forecasting and Prediction?, Journal of Forecasting , J. Forecast. 17, 481-495, (1998).

14. Kurbatsky V.G. Automation and telemechanics., No. 5., 143-158. (2014).

15. Al Zichri Balasim Mohammed. Increase in the accuracy of short-term forecasting of electric load of consumers in the region, taking into account metofactors on the basis of the support vector method: dis. Cand. tech. Sciences: 05.14.02, Al Zichieri Balasim Mohammed; Novocherkassk. YURPU (NPI), (2015)

16. A.S. Gordeev, Issues of modern science and practice. No. 2 (12), Volume 2, 33-36, (2008).

17. B. Makoklyuev Analysis and planning of power consumption, (M.: Energoatomizdat, 2008). 